\title{
Germanica
}

GERMANICA

36 | 2005

Le pouvoir de la musique dans l'espace de langue allemande : fascination et suspicion

\section{Une nouvelle grande invasion ou le jazz dans l'opéra de Krenek Jonny spielt auf}

Eine neue Völkerwanderung oder Kreneks Jazzoper Jonny spielt auf

\section{Pascale Avenel-Cohen}

\section{OpenEdition}

\section{Journals}

Édition électronique

URL : http://journals.openedition.org/germanica/1514

DOI : 10.4000/germanica.1514

ISSN : 2107-0784

Éditeur

Université de Lille

Édition imprimée

Date de publication : 30 juin 2005

Pagination : 67-79

ISBN : 9782913857155

ISSN : 0984-2632

Référence électronique

Pascale Avenel-Cohen, « Une nouvelle grande invasion ou le jazz dans l'opéra de Krenek Jonny spielt auf», Germanica [En ligne], 36 | 2005, mis en ligne le 10 juillet 2012, consulté le 06 octobre 2020. URL http://journals.openedition.org/germanica/1514 ; DOI : https://doi.org/10.4000/germanica.1514

Ce document a été généré automatiquement le 6 octobre 2020.

(c) Tous droits réservés 


\title{
Une nouvelle grande invasion ou le jazz dans l'opéra de Krenek Jonny spielt auf
}

\author{
Eine neue Völkerwanderung oder Kreneks Jazzoper Jonny spielt auf
}

\author{
Pascale Avenel-Cohen
}

1 En 1927 et 1928 l'actualité musicale allemande est dominée par une seule et unique production : l'opéra jazz de Ernst Krenek Jonny spielt auf, crée à Leipzig en 1927 et repris dans l'année qui suit sur vingt-cinq scènes allemandes dans des mises en scènes variées. Que ce soit pour la décrier ou l'encenser, aucun des acteurs de la vie culturelle ne reste insensible à cette intrusion du jazz dans les nobles maisons de ce que d'aucuns considèrent comme un genre compassé qu'il est temps de renouveler, alors qu'il constitue un art intouchable et pérenne pour les autres. Il est vrai qu'avec le succès croissant des théâtres de variétés, revues et autres spectacles musicaux dont l'essor est considérable dans ces années 20 , le débat sur le statut et la forme de l'opéra fait rage dans la presse musicale. Il s'inscrit d'ailleurs dans une perspective plus large ou la musique néo-objectiviste incarnée par Hindemith, et la musique moderne en général, que Stravinsky représente alors au niveau international, s'opposent à la tradition néoclassique "romantique ». Ce débat sur l'opéra, forme ancienne ou nouvelle, est celui d'un art élitiste opposé à un art populaire et démocratique. Mais le cas de Jonny dépasse ce simple clivage. Le ton passionné des réactions s'explique pour une autre raison : non seulement le jazz fait partie intégrante de la musique, mais Jonny met en scène un Noir triomphant de la culture occidentale, ou plutôt un " Nègre " dans le vocabulaire de cette époque. Krenek ne s'est donc pas contenté d'une incartade commerciale : il fait du triomphe du jazz sur la musique allemande, non seulement la pratique, mais aussi la théorie ${ }^{1}$. Car dès ses premières apparitions les nationalistes allemands ont condamné le jazz comme une « musique de Nègres » et n'ont jamais revu leur jugement.

2 Dans ce contexte, il n'est pas surprenant que les nazis aient mené croisade contre le spectacle avec leurs méthodes habituelles: campagne de dénigrement par voie 
d'affiches ${ }^{2}$, bousculades et manifestations dans les files d'attente, boules puantes, poil à gratter dans les salles ${ }^{3} .$.

3 Ils ne sont pas les seuls à s'insurger contre ce prétendu abâtardissement de l'art musical allemand le plus noble. La majorité des détracteurs de Jonny se recrute bien entendu parmi les milieux conservateurs et xénophobes, dans des périodiques comme Der Kunstwart, die Allgemeine Musikzeitung, Zeitschrift für Musik. Krenek est accusé d'un double sacrilège : esthétique, parce qu'il remet en question un art majeur, et politique, parce qu'il mettrait en péril la civilisation européenne, sachant qu'à cette époque peutêtre plus qu'à toute autre, politique et esthétique ne font qu'un en Allemagne.

Le livret de Krenek soulève d'emblée des objections véhémentes pour les tenants d'une esthétique européenne supérieure, que ce soit face aux États-Unis modernes ou aux Afro-Américains, doublement condamnés : à la fois comme membres d'une société dominée par la mécanique déshumanisée et comme représentants d'un stade prétendu antérieur de l'humanité. Un rappel de l'argument permettra au lecteur de mieux appréhender les raisons de ces réactions extrêmes ainsi que le malentendu entre le compositeur et ses spectateurs et critiques, malentendu dont Krenek lui-même est largement responsable. La complexité de l'intrigue nécessite un résumé assez détaillé : $(I, 1)$ Près d'un glacier, le compositeur Max rencontre par hasard la chanteuse Anita effrayée par la solitude glacée. Elle le prie de la ramener à son hôtel; il consent à quitter provisoirement cette nature adorée. (I.2) Ils s'aiment, mais Anita s'ennuie tandis que Max souhaite la garder pour toujours auprès de lui. Bien qu'il lui ait offert le manuscrit de son nouvel opéra, il lui demande de renoncer à le chanter. Elle part tout de même (I.3) Dans l'hôtel à Paris joue un jazz band ; Jonny, jazzman amant d'Yvonne, la femme de chambre, voudrait pénétrer dans la chambre de Daniello, violoniste virtuose et coureur de jupons, propriétaire d'un violon Amati. Alors qu'elle revient de l'opéra et se prépare à retourner vers Max, Anita est agressée par Jonny. Daniello la sauve avant de la séduire et de passer la nuit avec elle. Pendant ce temps, Jonny dérobe le précieux violon et le dissimule dans la boîte du banjo d'Anita. (I.4) Le lendemain matin, Anita s'apprête à rentrer chez Max. Désirant se venger de cette rupture, Daniello lui demande une bague en souvenir. C'est alors qu'il découvre le vol de son violon. Renvoyée, Yvonne est aussitôt engagée par Anita. Daniello donne la bague à Yvonne et lui ordonne de la remettre secrètement à Max. ( $2^{e}$ partie, scène 5) Max attend Anita toute la nuit et se plaint de ne plus puiser dans le glacier la force d'antan. (II.6) Mais il rêve qu'Anita lui revient. Alors qu'elle apparaît enfin au matin, Max, qui trouve son équilibre grâce au glacier et Anita, qui considère que la vie et la joie se trouvent seulement dans le mouvement, confrontent leurs points de vue. Max, à qui Yvonne donne la bague d'Anita, se précipite vers le glacier. Pendant ce temps, Jonny rejoint Anita et reprend possession du violon. Il explique à Yvonne que le violon lui appartient puisque c'est la musique du Nouveau Monde qui recueille l'héritage du vieux monde. (II.7) Max, désespéré, tente de se suicider, mais le glacier le renvoie vers les hommes et lui enjoint de ne plus avoir peur de la vie. Il reprend courage en entendant Anita chanter sa chanson par les haut-parleurs de l'hôtel proche. En entendant à la radio le jazz band de Jonny, Daniello reconnaît le son de son violon et alerte les autorités. (II.8) Jonny est poursuivi par la police. (II.9) À la gare il dépose le violon dans les bagages de Max qui attend Anita pour l'accompagner aux États-Unis, où elle doit chanter. L'instrument découvert, Max est appréhendé. Daniello raconte à Anita ce qui vient de se passer avant de tomber sous le train en tentant d'empêcher Yvonne d'innocenter Max en dénonçant Jonny. (II.10) Jonny lui promet de libérer Max et s'empare de la 
voiture de police qui attend le compositeur. Max comprend pendant le trajet que sa passivité est seule responsable des événements en cours. Il décide alors d'agir et de retourner à la gare tandis que Jonny met hors de combat les policiers qui les escortent. (II.11) Au dernier moment, Max rejoint Anita dans le train et la convainc qu'il a changé. Jonny reste à la gare et, perché sur l'horloge qui s'est transformée en globe terrestre, joue du violon tandis que la foule s'abandonne volontiers à la musique du Nouveau Monde. Les paroles du chœur à ce moment sont sans ambiguïté: «Les heures marquaient le temps passé, un nouveau temps commence maintenant. Ne ratez pas la correspondance, le trajet commence au pays inconnu de la liberté. [...] Le trajet commence, et Jonny nous fait danser. Le nouveau monde vient par la mer, étincelant, et recueille l'héritage de la vieille Europe grâce à la danse ${ }^{4}$ ». D'ailleurs, même Daniello reconnaît la supériorité de Jonny.

En fait, la réaction hostile des milieux esthétiques conservateurs s'explique essentiellement par la personnalité de Krenek. Jusqu'à cette œuvre et, au demeurant, avec ses œuvres postérieures, il incarne pour certains le renouveau de la musique allemande de haut niveau, par opposition avec les néo-objectivistes tels Hindemith, qui souhaitent rendre la musique accessible au plus grand nombre. Avec ses pièces atonales ou abstraites, Krenek n'est pas un musicien grand public. Sans être pour autant confidentiel, il est surtout apprécié par les spécialistes. En créant pour ainsi dire le genre de l'opéra d'actualité (Zeitoper) et en cédant au goût le plus simple du public, il se déjuge auprès de ses plus fervents admirateurs. C'est le cas de Hans F. Schaub qui exprime toute sa perplexité dans sa recension de la production hambourgeoise de l'opéra ${ }^{5}$. Il ne parvient pas à s'expliquer qu'un excellent musicien, sur qui portent les espoirs des critiques quant à l'avenir de la musique allemande, puisse céder au chant des sirènes au point de sacrifier les fondements même de son art. Car, précise le critique, une telle association de la vie quotidienne ${ }^{6}$ et de l'art ne peut avoir que des conséquences funestes. Pour justifier un tel paradoxe, il préfère penser, et affirmer, que Jonny est une parodie et que l'œuvre dénoncerait justement les méfaits de cette "épidémie au quotidien", de "ce rut jazz d'une société dégénérée »" Malheureusement défendre ainsi Krenek contre lui-même s'avère une tâche bien difficile, car le discours du livret n'est pas même ambigu. Il affirme bien un triomphe, d'autant plus que le jazz joue un rôle majeur dans la musique de l'opéra. Schaub est contraint de l'admettre. Toutefois il préfère en rester à cette interprétation, sans quoi il serait obliger de concéder, que, non seulement la « vie » fait irruption dans les temples de la musique allemande, mais aussi et surtout le jazz. Et si Krenek, l'un des représentants le plus prometteurs de la musique nationale aux yeux de ces conservateurs xénophobes, cédait à cette épidémie ${ }^{8}$, alors cela signifierait pour eux que la culture allemande serait bel et bien en péril.

6 En effet, outre ce que les conservateurs considèrent comme du domaine du quotidien, par le biais de la danse à laquelle le jazz est systématiquement associé dans ces années, c'est avant tout la dimension noire américaine du jazz qui leur fait horreur et les effraie. De fait, à cette époque jazz et négritude sont quasi automatiquement associés, ce qui ne sera plus le cas quelques années plus tard, alors que les Européens et les Allemands se seront approprié cette musique au point souvent d'en nier les origines ${ }^{9} \mathrm{ou}$ tout au moins de dénier une authenticité supérieure au jazz afro-américain. Or, cette négritude relève tout particulièrement du fantasme dans une Allemagne où l'épisode 
colonial fut de courte durée. Le texte expressionniste de Klabund, intitulé laconiquement « Der Neger $»^{10}$ en est un bon exemple.

7 On y trouve à peu près tous les poncifs du genre poussés à leur paroxysme jusqu'au viol par le personnage de plusieurs vierges blondes comme hypnotisées qu'il précipite ensuite dans un ravin. D'une sexualité débridée, animal plus qu'il n'est homme, vivant dans la pourriture, il ensorcelle, plus qu'il ne séduit véritablement l'héroïne, symboliquement nommée Yseult. Les xénophobes partagent alors tous cette vision de l'homme noir, attisée par une campagne haineuse au moment de l'occupation des bords du Rhin par les troupes françaises d'Afrique. Que les jazzmen noirs viennent d'une Amérique puritaine et non d'Afrique ne change strictement rien à ce point de vue. Pour ces racistes, "le Nègre", au singulier car il est considéré comme une catégorie indistincte, ne peut pas prétendre à incarner la civilisation dans la mesure où ils ne considèrent les Noirs au mieux que comme protohumains, c'est-à-dire restés au stade de l'enfance de l'humanité ${ }^{11}$.

8 Il est vrai que les rares Noirs avec lesquels les Allemands pouvaient entrer en contact étaient soit les Jazzmen arrivés après la première guerre mondiale, qui ne parlaient pas allemand, soit quelques malheureux Africains exhibés dans des zoos comme des animaux ${ }^{12}$. Il était donc facile de faire peur en prêtant à des hommes peu ou mal connus des mœurs bestiales. La France fut ainsi accusée par les nationalistes de vouloir salir la « race» allemande par le viol systématique des Allemandes par ces prétendues bêtes. Bien que la réalité de l'occupation ait prouvé que l'immense majorité des soldats africains n'agressaient personne, les écrits sur cette "honte noire " (Schwarze Schmach) se multiplièrent à l'époque et laissèrent des traces dans l'imaginaire des Allemands, même amateurs des jazzmen noirs. À en croire certains de ces écrits, entre les troupes françaises et la « musique de Nègres » (Niggermusik), une nouvelle vague de grandes invasions déferlait sur l'Allemagne.

9 Le personnage de Jonny correspond tout à fait à cette vision de l'homme noir, à commencer par sa sexualité13 qui, ici, ne parvient à s'effacer que devant sa cupidité, autre instinct immédiat. On retrouve les pires préjugés de la campagne anti-sénégalais non seulement dans la "gueule furieuse et d'une sensualité bestiale $»^{14}$ de Jonny, interrompu dans sa tentative de viol, mais aussi dans son discours. Tandis qu'Anita tente de plus en plus mollement de lui résister, désarmée par cette sensualité débridée qui la domine en même temps qu'elle la dégoûte, Jonny lui explique qu'il a déjà " séduit » de très nombreuses parisiennes, toutes très satisfaites de ses services. La preuve en est que, submergé par une pulsion aussi subite qu'irrépressible, il agresse Anita alors qu'il a une liaison avec Yvonne et que la femme de chambre se trouve à peine à quelques pas. La formule banale dans le discours xénophobe, mais non moins explicite de "diable noir $»^{15}$ lui est attribuée par Yvonne. Et, de fait, Jonny est diabolique, tant par la ruse qu'il emploie pour voler le violon d'abord et faire accuser un autre à sa place, que par son immoralité. Il ne recule devant aucun subterfuge et semble être un professionnel du crime. Mettre la police hors d'état de nuire, voler un véhicule, échapper à ses poursuivants, prendre Anita en filature pour retrouver le violon, tout ceci est un jeu d'enfant pour lui. Sans parler de la manière dont il ensorcelle tout le monde en jouant d'une musique qui, du simple fait d'un caractère rythmique très marqué à cette époque, est ressentie par un grand nombre de détracteurs comme lubrique, voire comme une véritable incitation au rut pour des hommes et des femmes qui perdent la maîtrise d'eux-mêmes, emportés par un rythme 
tribal ${ }^{16}$. Même s'il n'a pas l'étoffe d'un Méphistophélès, Jonny n'en est pas moins un grand séducteur, criminel et habile. Quoiqu'éculée depuis des siècles, l'association entre le noir et le diable continue à opérer. Krenek la reprend comme si elle était devenue une vérité.

Le jeu des associations hâtives ne s'arrête pas là : Jonny est noir, donc c'est un diable, sa sexualité est débridée et il se comporte comme un Africain.

11 Cette confusion habituelle à l'époque entre la couleur de la peau et l'Afrique, synonyme de primitivité, est également présente dans l'opéra: Au moment où il retrouve le violon, gagné par l'euphorie Jonny " danse en rond comme un Noir ${ }^{17}$ dans le salon de Max. Or les didascalies, toujours d'une précision extrême, ne donnent aucune indication sur cette manière de danser soit-disant typique, à croire qu'il s'agit d'une évidence et que tous les Noirs de la terre dansent de la même façon. La juxtaposition de ce comportement "primitif» avec les méthodes de gangster du personnage participe d'ailleurs de cet amalgame hâtif typique des années vingt.

Ce sont tous ces poncifs auxquels ils croient aveuglément et que Krenek leur livre complaisamment qui, concentrés dans le peu de scènes du personnage, font réagir avec tant de fougue les nationalistes ${ }^{18}$. Ils ne peuvent tolérer qu'un « sauvage », car c'est bien de cela qu'il s'agit, triomphe de la civilisation occidentale. Or, il est clair, que l'œuvre proclame la victoire du "nouveau monde" sur "l'ancien» comme le montrent les paroles mêmes de Jonny ${ }^{19}$ reprises à la fin par le chœur, alors que tous les voyageurs de la gare s'abandonnent au jazz, et que même Daniello, victime du vol et témoin de la scène admire le jeu de Jonny, désormais maître du violon Amati, puisque plus personne ne le lui conteste. Pourtant passé sous le train deux scènes plus tôt, Daniello est semblet-il ressuscité au baisser de rideau pour affirmer la victoire de Jonny avec d'autant plus de force qu'il en était la principale victime et en devient un admirateur inconditionnel dès lors qu'il l'entend jouer.

13 Ce triomphe d'un violeur et voleur noir est d'autant plus difficile à accepter pour les critiques nationalistes, que l'opéra lui-même le démontre puisque le jazz en est une composante non négligeable au point qu'on ait érigé Krenek, un peu hâtivement tout de même, en inventeur de l'opéra jazz. C'est ainsi que le compositeur est accusé de "salir la culture " par sa "sacralisation du Nègre ${ }^{20}$.

14 Car les nationalistes prêtent au jazz les mêmes caractéristiques qu'aux Noirs de leurs fantasmes : en tant que «musique de Nègres » ils perçoivent le jazz comme un appel constant à la luxure et la lubricité. Dans son article du Kunstwart, Eberlein parle ainsi à dessein de "Sexophone " à propos de l'instrument de Jonny ${ }^{21}$. Mais par-delà le goût du calembour, c'est tout le jazz qui est visé, car, avant que les percussions ne prennent la relève, pendant toutes ces années, le saxophone incarne pour beaucoup le jazz à luiseul. Métonymie du jazz sous forme de saxophone, il le devient tout naturellement sous forme de "sexophone" et contamine tout un genre musical. Ainsi, affirme ce même rédacteur, cette «métrique de Nègres» (Niggermetrik) colonise-t-elle toute l'Allemagne avant qu'on ne la retrouve sur le «tas d'ordures de toutes les drogues ${ }^{22}$. Envoûtés par la syncope, gagnés par l'américanisation jusque dans leurs plaisirs les plus simples, et autrefois les plus allemands, tels que la danse, les Allemands sont censés tourner le dos à leur culture nationale avec la plus parfaite insouciance... et indécence. En effet, en se livrant à ces exercices de gymnastique (Turnerei), les danseuses de Charleston, cette « danse de Nègres » ne montrent-elles pas leurs genoux, contribuant ainsi à la diffusion de l'obscénité dans la société allemande $!^{23}$ Les conservateurs de tous 
ordres regrettent la valse viennoise si sage et si allemande (sic) dont ils oublient au passage les relents d'indécence des débuts.

Les explications postérieures de Krenek pour relativiser l'importance du jazz et de la sacralisation d'un nouveau monde noir, porteur des valeurs d'avenir, expliquant que la figure centrale de l'œuvre n'est pas Jonny, mais le compositeur Max, ne convainquirent personne, d'autant que le titre indique le contraire. Il n'est pas surprenant dès lors que l'opéra ait été interdit par les nazis comme " art dégénéré ».

16 Mais la soi-disant sacralisation de la négritude, qui se trouve plutôt en être la dénaturation par le biais des préjugés racistes les plus ordinaires, n'est pas le seul sacrilège qu'ait commis Krenek dans son opéra. Il est aussi accusé de dégrader l'art en l'abaissant au niveau du quotidien. Bref il l'insère dans la société et dans l'actualité. D'atemporel, l'art devient prisonnier d'une période extrêmement datée. Et, de fait, passé son succès initial, l'œuvre n'a plus été beaucoup jouée ${ }^{24}$.

Le jazz participe pour une large part de cette dimension quotidienne, non plus en tant que « musique de Nègres » mais simplement en tant que musique de danse. Car, à cette époque et pour la grande majorité des Européens, amateurs ou détracteurs du jazz, quel que soit leur niveau social et leur horizon politique, cette musique est synonyme de danse, ce que certains déplorent. Malgré quelques tentatives de critiques pour rappeler qu'après tout, le menuet, élément constitutif s'il en faut de la grande musique, n'était au départ qu'une danse populaire ${ }^{25}$, les conservateurs rejettent cet argument. Ils refusent de mettre sur un même pied les danses du Chevalier à la rose et celles de Jonny, comme se plaisent à le faire les rédacteurs de la revue viennoise moderniste Musikblätter des Anbruchs ${ }^{26}$. Ils voient dans la danse au son du jazz le signe d'une ivresse populaire, voire d'une épidémie. D'ailleurs, dans l'opéra, lorsque la radio diffuse du jazz, les clients de la terrasse de montagne sont saisis de "mouvements rythmiques" irrépressibles et se trémoussent sur leurs chaises avant de se mettre à danser, incapables de résister à l'appel du saxophone ${ }^{27}$. Les amateurs ne sont d'ailleurs pas loin de partager ce point de vue et l'ivresse jazz (Jazzrausch) devient vite un topos dans les années vingt. La communion dans la danse jazz de tous les personnages et de tous les anonymes qui se trouvent dans la gare lorsque Jonny se met à jouer du violon ne fait que renforcer cette impression.

18 Il faut tout de même préciser au lecteur d'aujourd'hui que, lors de leur apparition en Allemagne après la Première Guerre mondiale, les danses jazz révolutionnent les bals et les salons des grands hôtels. Il n'est pas un orchestre de village qui ne se mette à la nouvelle mode, et les danseurs s'agitent sur des rythmes syncopés, pas toujours bien maîtrisés par les musiciens ${ }^{28}$, il faut bien le dire.

19 S'agiter est d'ailleurs le terme adéquat pour des danses telles que le shimmy, qui fit fureur dans les années 1920-1922 avant d'être supplanté par le fox-trot, le charleston ou, un temps par le black-bottom. Caractérisé par un tressaillement des épaules, le shimmy peut avoir dérouté des habitués de la valse et donné l'impression qu'il s'agissait plus d'un sport étrange que véritablement d'une danse. L'aspect sportif de la plupart de ses danses, pour lesquelles une bonne condition physique n'était pas superflue, est l'un des arguments essentiels des détracteurs du jazz en temps que genre noble. En outre, le caractère, certes très précis des pas pour chaque danseur, mais désordonné à l'échelle de toute une salle de bal, des déhanchements et mouvements des pieds, des mains et des épaules ne s'accordait certes pas avec l'idéal grec d'harmonie revendiqué par l'humanisme. Mais après quatre ans de guerre, beaucoup 
commençaient à douter des vertus de l'humanisme allemand et trouvaient dans la danse un exutoire autant qu'un plaisir évident, immédiat, simple. Si, pour les uns, l'art tombe dans le caniveau en sortant de sa tour d'ivoire et la culture se fait bien de consommation, pour les autres, faire du jazz un objet quotidien est une libération : elle redonne au corps une place naturelle à côté de l'esprit, loin de la « dignité » prussienne et belliqueuse, une place qu'il avait perdu et qu'il est grand temps qu'il retrouve pour le bien-être de tous et pour la paix ${ }^{29}$.

C'est cette simplicité qui révulse les opposants au jazz en tant que " musique utilitaire " (Gebrauchsmusik), formule revendiquée par les tenants de la Nouvelle Objectivité. Ils ne comprennent pas qu'un compositeur allemand puisse oser porter à la scène la même musique que dans «les bars, au cinéma, dans les salles de danses, les bistrots et les bordels $\aleph^{30}$. À double titre, ils accusent donc Krenek de souiller l'art allemand ${ }^{31}$.

La dimension quotidienne et actuelle de l'œuvre ne se limite toutefois pas au jazz, entaché par ses origines afro-américaines. Krenek la revendique aussi en faisant entrer sur une scène d'opéra des accessoires nouveaux, typiques de la vie trépidante des grandes villes modernes : non seulement le train, mais aussi le klaxon des automobiles, les sirènes de la police, le téléphone, la radio, des haut-parleurs, les néons publicitaires, le signal d'alarme de la gare, la sonnerie de départ du train autant d'éléments d'une grande modernité en $1927^{32}$ que la plupart des critiques refusent pour des raisons diverses. S'agissant des conservateurs, ils y voient bien sûr le symptôme d'une contamination de la scène autrefois noble de l'opéra par des éléments qui lui sont étrangers. Eberlein, toujours aussi haineux, force le trait jusqu'à parler d'un épisode de Vaudeville «transformé par le jazz en une nuit de Valpurgis avec automobile, train, téléphone et autres accessoires ${ }^{33}$. Pourtant, ces malheureux accessoires ne sont rien en regard des mises en scène de Piscator ou, à peine plus tard de Kurt Weill, qui n'hésitent pas à utiliser aussi des moyens techniques modernes sur scène, tels que le cinéma. Mais il est vrai que les nationalistes n'espéraient rien ni de l'un ni de l'autre, et déversent leur fiel sur eux avec moins de ressentiment que sur Krenek.

Peut-être plus que par une sirène de police, ils auraient dû être troublés par le tempo $\mathrm{du}$ deuxième acte dont les accessoires de la vie courante ne sont qu'un avatar. Il s'agit d'une double course poursuite, de Jonny pour échapper à la police et récupérer le violon, et de Max pour échapper lui aussi à la police qui l'a interpellé par méprise et réussir à rejoindre Anita avant qu'elle ne quitte l'Europe pour toujours. Au moins autant que le téléphone, ce rythme est un signe de ce "nouveau temps » venu d'Amérique salué par le chœur final comme celui du renouveau et de la liberté. "L'homme nouveau est pressé » résumait Stuckenschmidt ${ }^{34}$. Beaucoup de contemporains déplorent le rythme de vie de cette société nouvelle, sans cesse pressée, sans cesse à la poursuite de quelque chose, et symbolisée ici par la grande horloge de la gare et par le chœur des voyageurs: "Au plaisir! Aux affaires! Cela va bien trop lentement avec le chemin de fer » affirment-ils en passant ${ }^{35}$. Or, outre ces accessoires et ces jeux de lumières incongrus pour certains, inadmissibles pour les autres, ce tempo est aussi celui du jazz par le biais de la syncope ${ }^{36}$. D'ailleurs, l'Amérique tant prisée n'est incarnée sur scène que par Jonny, le jazzman noir, qui se languit du "swanee River " connu surtout en Allemagne à l'époque par le blues du même nom. Et la sacralisation du temps nouveau par le chœur est concomitante à son ode au jazz. Ainsi, même si on ne peut pas limiter l'accélération du rythme de vie des années vingt au jazz, il y participe largement, sonnant le glas d'une époque dont nombre de nostalgique rêvaient 
comme d'un âge d'or où la douceur de vivre aurait prévalu, oubliant un peu vite que cette période s'est terminée en 1918 par quatre ans de carnage.

Pourtant, si les nationalistes ou conservateurs voient en Jonny une intrusion insupportable de la modernité la plus extrême, les tenants d'une musique et d'une société moderne déplorent au contraire le conservatisme à peine déguisé de l'œuvre et la méconnaissance du jazz dont fait preuve Krenek. Là où les uns crient au sacrilège, les autres regrettent un manque d'audace manifeste. En effet, l'esthétique d'un " postromantisme puccinien $»^{38}$ de l'œuvre, en particulier des scènes de Max, n'est pas contrebalancée par un jazz somme toute très arrangé, voire germanisé. On est bien loin de la spontanéité d'un vrai jazz band noir, d'ailleurs Krenek a plutôt puisé son inspiration dans l'orchestre de « jazz symphonique » de Paul Whiteman.

Sa méconnaissance se montre au grand jour lorsqu'il fait jouer à Jonny pas moins de quatre instruments de technique radicalement différentes : saxophone, violon, banjo et trombone ${ }^{39}$, laissant peu de possibilités aux autres membres de l'ensemble. Pour les critiques modernes qui appellent de leurs vœux un art démocratique en prise sur la réalité de son temps, le jazz et les quelques accessoires sonores (téléphone, sirène...) auxquels recourt Krenek s'avèrent d'une telle superficialité qu'ils ne peuvent en aucun cas restituer l'essence de la société contemporaine. Ils expliquent le succès de l'opéra autant par le pseudo jazz de Jonny que par l'esthétique romantique du dix-neuvième siècle qui, finalement, ne dépayse pas le public profane. En un mot, ils reprochent son conformisme et sa complaisance à Krenek $^{40}$ et explique le succès fracassant de l'œuvre dès sa création à Leipzig par le talent du metteur en scène Walther Brügmann ${ }^{41}$.

En fait d'invasion barbare, le premier opéra jazz écrit par Krenek resta aussi le dernier. Les autres « opéras d'actualité » de cette époque sont ceux de Kurt Weill qui, bien qu'il utilise aussi les éléments du jazz, ne le revendique pas de manière aussi ostentatoire et sait créer un style personnel. Bien que les détracteurs de l'opéra prétendent justifier leur position par des arguments esthétiques, il est évident que la politique seule dicte leurs paroles. Le « jazz » qui les dérange est bien peu subversif, quant au personnage de Jonny qu'ils abhorrent, il ne fait que renvoyer l'image qu'ils ont fabriquée d'un homme noir immoral et séducteur, comme le diable, dont il est censé avoir la couleur. En reprenant ces clichés racistes et en composant un jazz stéréotypé, Krenek s'est complaisamment offert en pâture à leurs critiques, même s'il en a paru surpris et blessé. De fait, Jonny spielt auf est bien un " opéra d'actualité ", dans la mesure où il nous informe sur la vision que la plupart des Allemands de cette époque avait des jazzmen, du jazz et de sa symbolique politique et sur la séduction qu'exerçait alors cette musique sur des hommes et des femmes en quête de joie de vivre et de simplicité.

\section{NOTES}

1. «In praxi und theoria », cf. article annonyme « Krenek, der Freudenbringer », dans Zeitschrift für Musik, 1927, H.11 p. 641. 
2. L'appel à manifestation du NSDAP contre la production de l'opéra au Staatstheater de Vienne en 1928 en est un exemple, cf. livret de l'enregistrement de la production de l'opéra à Leizig en 1990, Decca 436 631-2, p. 49.

3. Michel Collomb : «Le tumulte noir. Joséphine Baker à Berlin et à Vienne », dans Cahier d'Études Germaniques, 1993/24, p. 133-134.

4. Livret p. 254.

5. Hans F. Schaub : «Kreneks Jonny im Hamburger Stadttheater ", dans Allgemeine Musikzeitung, 1927, H.26, p. 731-732.

6. « Anschluß an das Leben » id. p. 732.

7. «Sollte es eine Persiflage der Alltagspest, der Jazzbrünste einer degenerierten Gesellschaft sein ?»

8. Il est intéressant de signaler que même les plus fervents défenseurs du jazz le présentent comme une épidémie, une sorte d'ivresse universelle, dont ils saluent les effets libérateurs sur les corps et les esprits contraints pendant des siècles par le cadre beaucoup trop étroit de l'idéalisme. Cf. par exemple l'emploi de l'expression «Tanzepidemie » par Herwarth Walden : «Die Tanzhalle des Berliner Westens », dans Der Sturm, 1925, H.2, p. 31-32.

9. C'est ainsi que le jazz est présenté comme «entièrement l'affaire des Blancs » («obwohl der Jazz eine durchaus ,weiße' Sache ist») dans l'article de Kurt Heuser: "vom afrikanischen Neger », dans Die Neue Rundschau, 1930, vol. 1, p. 834-844.

10. Klabund, Der Neger, 1920, Dresden, Kaemmerer Verlag, 1920, 22 pages. Ce texte ne figure pas dans la plupart des oeuvres complètes.

11. Il est à déplorer que beaucoup des amateurs du jazz contribuent à diffuser ces préjugés en accréditant la thèse du bon sauvage, certes bon, mais néanmoins sauvage, eu égard aux AfroAméricains.

12. Balder Olden : « Ballade aus dem Zoo », dans Das Tagebuch, 1925, H.25, p. 1058-1060.

13. Ainsi, alors que Jonny rompt son contrat, le directeur de l'hôtel s'affole et lui demande s'il veut plus de femmes (Weiber) ou plus d'argent, p. 150.

14. «Jonnys tierisch-sinnlich-wütende Fratze verwandelt sich in ein breites Grinsen », $1^{\text {re }}$ partie, scène 3 p. 116.

15. « schwarzer Teufel » id., p. 118.

16. Cf. «Produkt tierischer Brunst » ou « strotzend von Geilheit » dans Prof. Max Chop : « Jonny spielt auf », dans Signale für die musikalische Welt, 1927, Nr.43, p. 1477-1480, ici p. 1478.

17. « Wobei er eben wie ein Neger herumtanzt », $2^{\mathrm{e}}$ partie, scène 6, p. 192.

18. Cet aspect des choses ne signifie pas pour autant que Krenek ait voulu écrire un opéra raciste, mais il a repris le stéréotype en vigueur à l'époque sans trop se poser de questions.

19. «Mir gehört alles, was gut ist in der neuen Welt. Die alte Welt hat es erzeugt, sie weiß damit nichts mehr zu tun. Da kommt die neue Welt übers Meer gefahren mit Glanz und erbt das alte Europa durch den Tanz. ", $2^{\mathrm{e}}$ partie, scène 6, p. 194.

20. « Kulturschädiger », « Niggerverherrlichung » dans Paul Schwers : «Jonny spielt auf », dans Allgemeine Musikzeitung, 1927, H.7, p. 151-152.

21. Eberlein : « Johnny spielt auf », dans Der Kunstwart, 1928, H.4, p. 273-274.

22. «Aber ich sage, daß die Gesellschaft, aller Kreise durch diese Niggermetrik eines entgeisteten Bedeutungsaktes verdummt, typisiert und in eine Preßform getanzt wird, die man später einmal auf dem Müllhaufen aller Rauschmittel wiederfinden kann. », id., p. 273-274.

23. Richard H. Stein-Berlin : «Auslandspropaganda », dans Die Musik, 1927, H. 9, p. 639-645.

24. L'opéra a été monté plusieurs fois depuis 1958, sur trois scènes d'Autriche et de Suède en 1980 et à Leipzig en 1990. Cf. « Jonny spielt auf. Un 'Zeitoper' entre jazz et musique nouvelle », auteur anonyme, livret p. 41.

25. Hermann Schildberger : «Jazz-Musik », dans Die Musik, 1925, H.12, p. 914-923. 
26. Dr. Adolf Aber : « Ernst Krenek, Johnny spielt auf », dans Musikblätter des Anbruchs, 1927, H.3, p. 127-132.

27. $2^{\mathrm{e}}$ partie, scène 7, p. 208.

28. Cornelius Partsch : Schräge Töne. Jazz und Unterhaltungsmusik in der Kultur der Weimarer Republik, Stuttgart, Metzler, 2000, p. 59-60.

29. Cf. Hans Siemsen : « Jazz-Band », dans Die Weltbühne, 1921, H.10, p. 287-288.

30. Cf. note 20 p. 151.

31. " Es gibt immerhin auch heute noch einige charakterfeste Bühnenleiter, die lieber auf dieses Geschäft verzichten, als sich von der Schmutzwelle der Jonnyhauses ihr kulturellen Zwecken geweihtes Haus verunreinigen zu lassen » dans une note de la rédaction adjointe à l'article de Wilhelm Matthes : «Schwarze Schmach » dans Allgemeine Musikzeitung, 1927, H. 41, p. 1011.

32. Max téléphone à la gare (II, 5) p. 164, les haut-parleurs du palace de montagne diffusent la radio (II, 7) p. 204, la locomotive entre en gare sur scène dans un fracas épouvantable (II, 9) p. 238, la voiture de police conduite à toute allure par Jonny, sirène hurlante traverse une grande ville dont on voit la lumière des néons publicitaires (II, 10) p. 246, après un virage à $90^{\circ}$, Jonny braque les phares de la voiture sur le public, au ras du bord de la scène p. 248, enfin, à la gare (II, 11), la sonnerie de départ du train retentit de manière prolongée p. 250 et Jonny se dirige vers le signal d'alarme p. 252.

33. «[...] wenn jemals ein widerliches Intermezzo eines Weibes, revuemäßig garniert und frisiert, in eine moderne Walpurgisnacht mit Auto, Eisenbahn, Telephon und anderen Kulturrequisiten verjazzt wurde [...] », cf. note 21, p. 274.

34. Cf. Pascal Huynh : La Musique sous la République de Weimar, Fayard, collection les chemins de la musique, 1998, p. 250.

35. Seconde partie, scène 9, p. 220.

36. Pascale Avenel-Cohen : "Jazz de Hans Janowitz ou l'ère de la syncope », dans Cahiers d'Études Germaniques, 2004/1, ${ }^{\circ} 46$, p. 129-141.

37. $2^{\mathrm{e}}$ partie, scène 8, p. 214.

38. Cf. note 34, Pascal Huynh : La Musique sous la République de Weimar, p. 250.

39. Livret, p. 104.

40. « Meloskritik : “Jonny spielt auf” ", dans Melos, 1928, H.1, p. 24-25.

41. Heinrich Strobel : "Umschau: Ernst Krenek "Jonny spielt auf” ", dans Melos, 1927, H.3, p. 130-131.

\section{RÉSUMÉS}

Dès sa création en 1928, l'opéra jazz de Ernst Krenek Jonny spielt auf connut un très vif succès aussi bien en Allemagne qu'en Autriche. Face à l'engouement du public, les critiques restèrent assez froids dans leur majorité : Les Modernes déploraient le conformisme et le manque d'audace du compositeur dont le jazz manquait à leur yeux de spontanéité et de dynamisme alors que le reste de la musique restait trop prisonnier d'une esthétique du $\mathrm{XIX}^{\mathrm{e}}$ siècle. Les Anciens pour leur part refusaient que le jazz fasse intrusion dans un genre noble par excellence. Mais ce sont les nationalistes qui réagirent avec le plus de virulence. Ils ne supportaient ni l'utilisation par un compositeur allemand d'une «musique de Nègres » ni le message de l'opéra. En effet, Krenek y proclame à plusieurs reprises le triomphe de la culture américaine sur l'Europe, alors même que 
les États-Unis sont incarnés par un musicien de jazz noir. Or, le personnage de Jonny concentre tous les fantasmes racistes de l'époque : c'est un ensorceleur, un voleur, un violeur... autant d'éléments qui, de l'obscénité à un attrait irrésistible, contaminent tout un genre musical. Le virulent débat qui s'engage autour de cette œuvre est révélateur de l'approche politique du jazz, si caractéristique de la République de Weimar.

Von ihrer Erstaufführung 1928 an hatte Ernst Kreneks Jazzoper Jonny spielt auf einen großen Erfolg sowohl in Deutschland wie in Österreich. Dem Enthusiasmus des Publikums entgegen blieben die meisten Kritiker skeptisch : die Befürworter der Moderne warfen dem Komponisten seinen Konformismus vor : sein Jazz war ihnen nicht spontan und dynamisch genug, während die restliche Musik in einer Ästhetik des 19. Jahrhunderts verfangen blieb. Die Konservativen lehnten ihrerseits die Verwendung des Jazz in einer edlen Gattung par Excellence ab. Am heftigsten reagierten aber die Nationalisten. Sie konnten nicht annehmen, dass ein deutscher Komponist „Niggermusik“ gebrauchte. Auch die Botschaft der Oper war ihnen zuwider. In der Tat gibt Krenek mehrmals den Triumph der amerikanischen Kultur über Europa mehrmals kund, wobei die Vereinigten Staaten von einem schwarzen Jazzmann verkörpert werden. Außerdem konzentriert die Figur von Jonny alle damaligen rassistischen Wahnvorstellungen : ein Dieb, ein Vergewaltiger, der seine Opfer verhext... Eigenschaften die, von der Obszönität bis zum unwiderstehlichen Reiz eine ganze musikalische Gattung verseuchen. Diese heftige Debatte um Kreneks Oper ist typisch für die politische Rezeption des Jazz, die die Weimarer Republik kennzeichnet.

\section{AUTEUR}

\section{PASCALE AVENEL-COHEN}

Université Charles-de-Gaulle - Lille 3 